\title{
Green Tea Lozenge
}

National Cancer Institute

\section{Source}

National Cancer Institute. Green Tea Lozenge. NCI Thesaurus. Code C67058.

A lozenge preparation of green tea, derived from the dried leaves of Camellia sinensis, with potential antioxidant and chemopreventive activities. Green tea lozenge contains polyphenols that are believed to be responsible for its chemopreventive effect. The polyphenol fraction contains mainly Epig allocatechin-3-gallate (EGCG) and other catechins, such as epicatechin (EC), gallocatechin gallate (GCG), epigallocatechin (EGC), and epicatechin gallate (ECG). Green tea polyphenols act as antioxidants and free radical scavengers, thereby protecting cells from reactive oxygen species and further damage. 